Pure and Applied Mathematics Quarterly

Volume 6, Number 2

(Special Issue: In honor of

Michael Atiyah and Isadore Singer)

295-306, 2010

\title{
Spin Cobordism Categories in Low Dimensions
}

\author{
Jack Morava and Nitu Kitchloo \\ For Michael Atiyah, in deep gratitude.
}

\begin{abstract}
The Madsen-Tillmann spectra defined by categories of threeand four-dimensional Spin manifolds have a very rich algebraic structure, whose surface is scratched here.
\end{abstract}

Keywords: Spin cobordism, Madsen-Tillmann, topological gravity

\section{Cobordism Categories}

1.1 Many variations and generalizations are possible, but to begin, consider the topological two-category $D$ Cobord whose objects are oriented smooth closed $d$ manifolds $(D=d+1)$, with the topological category $D$ Cobord $\left(V, V^{\prime}\right)$ of morphisms having as objects, $D$-dimensional cobordisms

$$
W: V \rightarrow V^{\prime}
$$

from $V$ to $V^{\prime}$; for our purposes this will mean an identification $\partial W \cong V_{\text {op }} \amalg V^{\prime}$, extended to a small neighborhood of the boundary. The two-morphisms will be orientation-preserving diffeomorphisms of such cobordisms, which equal the identity near the boundary. The composition functor

$$
D \text { Cobord }\left(V, V^{\prime}\right) \times D \text { Cobord }\left(V^{\prime}, V^{\prime \prime}\right) \rightarrow D \text { Cobord }\left(V, V^{\prime \prime}\right)
$$

is defined by glueing outgoing to incoming boundaries.

Received November 9, 2006.

Both authors were supported by the NSF. 
A topological category $\mathbf{C}$ is a kind of simplicial space, and so has a geometric realization $|\mathbf{C}|$; for example,

$$
\mid D \text { Cobord }\left(V, V^{\prime}\right) \mid=\coprod_{\left[W: V \rightarrow V^{\prime}\right]} B \operatorname{Diff}_{0}^{+}(W)
$$

is the union, indexed by diffeomorphism classes of cobordisms $W$ from $V$ to $V^{\prime}$, of the classifying spaces of the groups of orientation-preserving diffeomorphisms of $W$ which equal the identity near the boundary. We'll write $D \mid$ Cobord $\mid$ for the topological category with closed $d$-manifolds as objects, and the classifying spaces above as morphism objects; it is symmetric monoidal (under disjoint union).

Such categories have an impressive history $[3,16,17,21]$ in topology and physics. This paper applies the recent breakthroughs of [9] which (in great generality) identify the classifying spectra of such categories. The formalism of Galatius, Madsen, Tillmann, and Weiss frames these categories somewhat differently: they work with a category $\mathcal{C}_{D}$ of manifolds embedded in a high-dimensional Euclidean background, but the description used above is equivalent, and is convenient in physics.

1.2 A topological transformation group $G \times X \rightarrow X$ has an associated homotopyto-geometric quotient map

$$
X / / G:=E G \times_{G} X \rightarrow \operatorname{pt} \times_{G} X=X / G
$$

which defines a kind of resolution

$$
B \text { Diff } \sim E \text { Diff } \times_{\text {Diff }} \text { Metrics } \rightarrow \text { pt } \times_{\text {Diff Metrics }}
$$

of the moduli space of Riemannian metrics [8] on a manifold. For a closed manifold the action of the diffeomorphism group on the space of metrics is proper; for surfaces of genus $>1$, for example, its isotropy groups are not just compact but finite, making the map a rational homology equivalence.

This resolution defines a monoidal functor

$$
D \mid \text { Cobord } \mid \rightarrow \text { Gravity }_{D}
$$

to a topological category with moduli spaces of metrics as its morphism objects. The Einstein-Hilbert functional

$$
g \mapsto \int_{W} R(g) d \operatorname{vol}_{g}: \text { Metrics/Diff } \rightarrow \mathbb{R}
$$


is a natural candidate for a Morse function on these objects, so this category models interesting aspects of (Euclidean) general relativity. Witten has suggested that backgrounding the choice of Morse function leads to more general models in which topology change can be treated quite naturally.

1.3.1 This paper is concerned with the cobordism category defined by fourdimensional Spin manifolds. The classifying space $|\mathbf{C}|$ of a symmetric monoidal topological category $\mathbf{C}$ is a kind of abelian monoid, or better: a $\Gamma$-space in the sense of [20]. Its group completion

$$
|\mathbf{C}|^{+}:=\Omega B|\mathbf{C}|
$$

is an infinite loop-space, which is characterized by its associated stable spectrum.

GMTW identify $|D|$ Cobord $\|\left.\right|^{+}$as the infinite loopspace associated to a twisted desuspension

$$
\operatorname{MTSO}(D):=B \mathrm{SO}(D)^{-\mathbf{D}}
$$

of the classifying space for the orthogonal group, where $\mathbf{D}$ is the vector bundle associated to the basic representation of $\mathrm{SO}(D)$ on $\mathbb{R}^{D}$. More generally, a pullback diagram

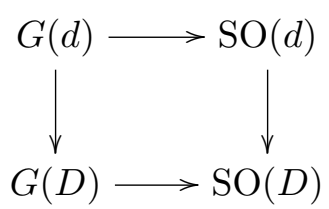

of groups and homomorphisms defines a topological category $G \mid$ Cobord| of manifolds with $G(d)$-structures on their tangent bundles, up to cobordism through manifolds with $G(D)$-structures; and the techniques of [9] identify its associated spectrum as

$$
M T G(D):=B G(D)^{-\mathbf{D}}
$$

where $\mathbf{D}$ is now the $D$-dimensional representation of $G(D)$ pulled back from the basic representation of the orthogonal group. A further generalization identifies the classifying spectrum for the category of $G$-manifolds mapped to some parameter space $X$ as

$$
X_{+} \wedge B G(D)^{-\mathbf{D}}
$$


1.3.2 When $d=1$, for example, we get the desuspension (alternately denoted $\left.\mathbb{C} P_{-1}^{\infty}\right)$ of $B \mathrm{SO}(2)=\mathbb{C} P^{\infty}$ by the tautological line bundle. Its homology is free of rank one in even dimensions $\geq-2$, and is otherwise zero. Pinching off its (-2)-dimensional cell defines a cofibration

$$
S^{-2} \rightarrow \mathbb{C} P_{-1}^{\infty} \rightarrow \mathbb{C} P_{+}^{\infty}
$$

of spectra, with an associated fibration

$$
\Omega^{\infty} S^{-2} \rightarrow|2| \text { Cobord } \|\left.\right|^{+} \rightarrow Q\left(\mathbb{C} P^{\infty}\right) \times Q\left(S^{0}\right)
$$

of loopspaces. Since $\Omega^{\infty} S^{-2}$ has torsion homotopy, the rational homology of $|2|$ Cobord ||$^{+}$is a free bicommutative Hopf algebra generated by $\tilde{H}_{*} \mathbb{C} P_{\infty}$ (ie, by the Miller-Morita-Mumford classes $\kappa_{i}, i \geq 1$ ), extended by degree zero classes $\kappa_{0}^{ \pm n}$ coming from the rational cohomology of $Q\left(S^{0}\right)$

1.3.3 The covariant functor $[12 \mathrm{Ch}$ III]

$$
X \mapsto \operatorname{Spec} H^{ \pm}\left(\Omega_{0}^{\infty} X, \mathbb{F}\right):=\tilde{\mathbf{H}}_{ \pm}(X, \mathbb{F})
$$

(from (Spectra) to unipotent commutative supergroup-schemes over the field $\mathbb{F}$ ) is a homotopy-theoretic analog of the 'big' quantum cohomology studied in some contexts in physics. For example, the infinite loopspace associated to the suspension spectrum $\Sigma^{\infty} X$ defined by a connected pointed space splits stably as

$$
\Omega^{\infty} \Sigma^{\infty} X \sim \coprod_{n \geq 0} E \Sigma_{n} \wedge \Sigma_{n} X^{\wedge n}
$$

so its rational cohomology is the symmetric algebra on the reduced cohomology of $X$. In this case $\tilde{\mathbf{H}}_{ \pm}(X, \mathbb{Q})$ can be identified with the affine (super)groupscheme which represents the functor

$$
(\mathbb{Q} \text { - algebras }) \ni A \mapsto \tilde{H}_{ \pm}(X ; A) \in(\mathbb{Q}-\text { Vect }) .
$$

For a general connected spectrum $X, H^{ \pm}\left(\Omega^{\infty} X, \mathbb{Q}\right)$ is the universal enveloping Hopf algebra associated to the (super-commutative) Lie algebra $\pi_{ \pm}(X) \otimes \mathbb{Q}$ of primitives. The category of such affine groupschemes is closed and symmetric monoidal, with a product $\otimes$ which is not very familiar $[12 \mathrm{Ch} \mathrm{II}]$; over $\mathbb{Q}$, it corresponds to the graded tensor product of spaces of primitives.

If the loop-space associated to a spectrum is not connected, let $\mathbf{H}^{0}(X, \mathbb{F})$ be the groupscheme represented by the group ring $\mathbb{F}\left[\pi_{0}(X)\right]$, and let $\mathbf{H}_{0}(X, \mathbb{F})$ be the 
spectrum of the ring of finitely-supported $\mathbb{F}$-valued functions on $\pi_{0}(X)$; then we can define

$$
\mathbf{H}_{ \pm}(X, \mathbb{F})=\mathbf{H}_{0}(X, \mathbb{F}) \times \tilde{\mathbf{H}}_{ \pm}(X, \mathbb{F})
$$

(and similarly, for cohomology). For example,

$$
\mathbf{H}_{ \pm}\left(S^{2} \times \operatorname{MTSO}(2), \mathbb{Q}\right)
$$

is an analog of the big quantum cohomology related to the Toda lattice [10].

\section{Low-Dimensional SPIn COBORDisms}

2.0 The action

$$
u, q \mapsto u q u^{-1}: \mathrm{SU}(2) \times \mathbb{H} \rightarrow \mathbb{H}
$$

of the group $\mathrm{SU}(2)=\{u \in \mathbb{H}|| u \mid=1\}$ of unit quaternions leaves the subspace $\mathbb{R} \subset \mathbb{H}$ invariant, defining a double cover

$$
\rho: \mathrm{SU}(2) \rightarrow \mathrm{SO}(3)
$$

of the rotation group of the subspace orthogonal to it, identifying $\mathrm{SU}(2)$ with Spin(3). Similarly, the action

$$
u_{L}, u_{R}, q \mapsto u_{L} q u_{R}^{-1}:(\mathrm{SU}(2) \times \mathrm{SU}(2)) \times \mathbb{H} \rightarrow \mathbb{H}
$$

factors through the double cover

$$
\mathrm{SU}(2) \times S U(2)=\operatorname{Spin}(4) \rightarrow \mathrm{SO}(4) .
$$

It is easy to see that the diagram

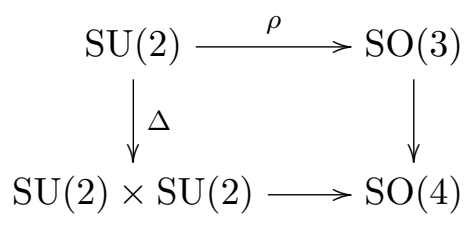

is a pullback; following $\S 1.3 .1$, this defines the cobordism category of Spin threemanifolds up to four-dimensional Spin cobordism. Similarly, the $D=3$ Spin cobordism category is defined by three-dimensional Spin cobordisms between two-dimensional Spin manifolds: in Riemann surface terms [2], the latter structure amounts to a choice of square root for the canonical complex line bundle. [Complex Spin structures are very interesting [22], but they won't be considered here.] 
We'll write $\mathbb{H}_{\mathrm{ad}}-1$ for the three-dimensional representation $\rho$, and $\mathbb{H} \otimes \mathbb{H}_{\mathrm{op}}$ for the four-dimensional Spin representation, as in $[13 \S 1.4,15 \S 1]$; then

$$
\operatorname{MTSpin}(3) \sim \Sigma B \mathrm{SU}(2)^{-\mathbb{H}_{\mathrm{ad}}}
$$

and

$$
M T \operatorname{Spin}(4) \sim B(\mathrm{SU}(2) \times \mathrm{SU}(2))^{-\mathbb{H} \otimes \mathbb{H}_{\mathrm{op}}}
$$

These spectra are very nice, with torsion-free integral homology concentrated in degrees $\equiv-1$ (resp. 0) mod four, but they are nontrivial in negative dimensions, starting in degree -3 (resp. -4). It will simplify notation below to introduce their connective suspensions

$$
\operatorname{MT}(3):=\Sigma^{3} M T \operatorname{Spin}(3)
$$

and

$$
\operatorname{MT}(4):=\Sigma^{4} M T \operatorname{Spin}(4)
$$

2.1 The representation $\mathbb{H} \otimes \mathbb{H}_{\mathrm{op}}$ restricts to $\mathbb{H}_{\text {ad }}$ along the diagonal embedding of $\mathrm{SU}(2)$ in $\mathrm{SU}(2) \times \mathrm{SU}(2)$. Since Thom spaces (and spectra) behave nicely under pullback, this defines a morphism

$$
\Delta_{\natural}: \operatorname{MT}(3) \rightarrow \operatorname{MT}(4) .
$$

The main result of this note asserts that (at least, up to cohomology) this map makes $\mathrm{MT}(3)$ a kind of cocommutative and coassociative coalgebra spectrum.

Proposition: The integral cohomology $H^{*} \mathrm{MT}(3)$ can be identified with $H^{*} B \mathrm{SU}(2)$ as an algebra, consistent with a splitting

$$
\Psi^{*}: H^{*} \mathrm{MT}(3) \otimes H^{*} \mathrm{MT}(3) \cong H^{*} \mathrm{MT}(4)
$$

which identifies $\Delta_{\natural}^{*}: H^{*} \mathrm{MT}(4) \rightarrow H^{*} \mathrm{MT}(3)$ with the multiplication map.

Proof: If $X$ is a compact connected space, then any $[V] \in \tilde{K} \mathrm{O}(X)$ is stably equivalent to a vector bundle $V$ over $X$, of dimension v $\gg 0$, and Atiyah's Thom spectrum

$$
X^{[V]}:=\Sigma^{-\mathrm{v}} X^{V}
$$


is well-defined up to homotopy. If $[V]$ is orientable (eg if $w_{1}(V)=0$, in the case of integral homology), there is a Thom isomorphism

$$
\Phi_{V}: H^{*} X \rightarrow H^{*} X^{[V]} .
$$

Taking a limit over finite subcomplexes extends such constructions to nice spaces like $B \mathrm{SU}(2)$.

With this notation, we have a commutative diagram

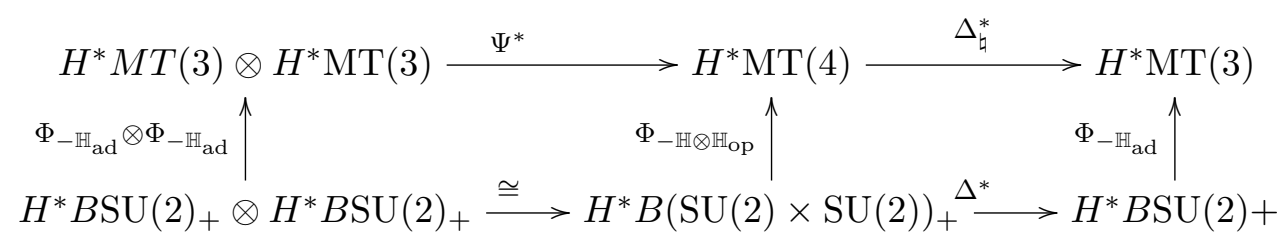

with the composition $\Delta_{\natural}^{*} \circ \Psi^{*}$ defining the multiplicative structure.

Verification of associativity amounts to unwinding the collection of Thom isomorphisms which reduce the commutativity (after taking cohomology) of the diagram

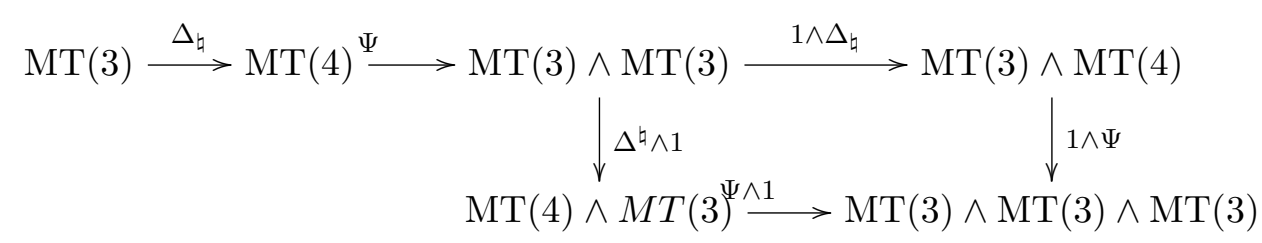

to a similar diagram expressing the associativity of the usual multiplication on $H^{*} B \mathrm{SU}(2)_{+}$. Commutativity is a consequence of $\Delta_{\natural}$ being essentially a diagonal, and the unit is the composition

$$
H^{*} B \mathrm{SU}(2)^{-\left[\mathbb{H}_{\mathrm{ad}}\right]} \stackrel{\Phi_{\mathbb{H}_{\mathrm{ad}}}^{-1}}{\longrightarrow} H^{*} B \mathrm{SU}(2)_{+} \longrightarrow H^{*} S^{0}
$$

defined by the inclusion of a basepoint into $B \mathrm{SU}(2)$. 
2.2.1 The result above can also be paraphrased in terms of a ring structure on homotopy quantum cohomology, but because

$$
\Omega^{\infty} \mathrm{MT}(3) \sim \mathbb{Z} \times \Omega_{0}^{\infty} \mathrm{MT}(3)
$$

is not connected, this requires some discussion. According to $\S 1.3 .1, \mathrm{MT}(3)$ is the cobordism spectrum of Spin three-manifolds mapped to the three-sphere. The extra data defined by such a map is (at least, after tensoring with $\mathbb{Q}$ ) very close to a framing (in the sense of $[4,15 \S 2.1]$ ) of a three-dimensional Spin cobordism.

Similarly,

$$
\Omega^{\infty} \operatorname{MTSpin}(4) \sim \mathbb{Z}^{2} \times \Omega^{\infty} M T \operatorname{Spin}(4)
$$

with the classes of a K3 surface and the quaternionic projective plane as natural geometric generators for $\pi_{0}$ [11]. The Euler characteristic $\chi$ and the signature $\sigma$ are a basis for the linear functionals on this group, at least over $\mathbb{Z}[1 / 2]$, and if $\chi^{*}, \sigma^{*}$ denote the dual basis elements, then

$$
\left[\begin{array}{c}
\mathbb{H} P_{2} \\
K 3
\end{array}\right]=\left[\begin{array}{cc}
2 & 0 \\
6 & 16
\end{array}\right]\left[\begin{array}{l}
\chi^{*} \\
\sigma^{*}
\end{array}\right]
$$

Desuspending the isomorphisms in $§ 2.1$ yields a splitting

$$
\mathbf{H}^{ \pm}(M T \operatorname{Spin}(4), \mathbb{Q}) \cong \otimes^{2}\left(\mathbf{H}_{K M}^{ \pm}\left(\Sigma^{-1} M T \operatorname{Spin}(3), \mathbb{Q}\right)\right)
$$

of homotopy-theoretic quantum cohomology: where the subscript on the right indicates an extension of the pro-unipotent object $\tilde{\mathbf{H}}^{ \pm}\left(\Sigma^{-1} M T \operatorname{Spin}(3), \mathbb{Q}\right)$ by the multiplicative group (represented by the group ring of Kirby-Melvin framings).

2.2.2 The existence of a multiplication on $H^{*} \mathrm{MT}(3)$ raises the possibility of the existence of a so-called Hopf algebroid structure on $\left(H^{*} \mathrm{MT}(3), H^{*} \mathrm{MT}(4)\right)$. In fact, two three-dimensional cobordisms mapped to the three-sphere define a fiber product

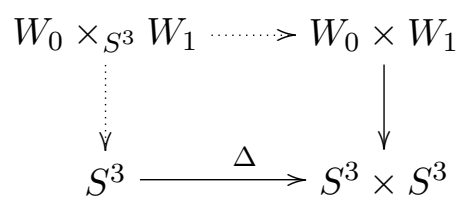


which is generically another such; but whether this can be used to define a geometric product on $\mathrm{MT}(3)$ involves subtle questions about framings.

Note that the symplectic pairing (and the associated duality) on the Tate cohomology $t_{\mathbb{T}} H \mathbb{Z}$ studied in connection with $\operatorname{MTSO}(2)$ in [18] has a very nice analog on $t_{\mathrm{SU}(2)} H \mathbb{Z}$.

\subsubsection{The spectrum}

$$
\operatorname{MTL}(4):=B \mathrm{SU}(2)^{-\mathbb{H}}
$$

(defined by the obvious action $\sigma$ of $\mathrm{SU}(2)$ by left multiplication on $\mathbb{H}$ ) is the Madsen-Tillmann spectrum of the category of three-dimensional Spin manifolds, up to cobordism through four-manifolds with an 'almost hyperHermitian' structure (in the sense of [7]). These cobordisms are essentially four-manifolds with $\mathrm{Sl}_{2}(\mathbb{C})$ (ie, Lorentzian Spin) structures; the cohomology of the associated spectrum is concentrated in even dimensions, but it has no very obvious multiplicative structure. It would be interesting to understand better the relations between this spectrum and MTSpin(3), which has cohomology concentrated in odd degrees: it is tempting to think of $M T \mathrm{~L}(4)$ as some kind of bosonization of $M T \operatorname{Spin}(3)$.

Behind this lie broader questions about Atiyah-twistings of spectra: the isomorphism

$$
H^{*} B \mathrm{SU}(2)^{-\mathbb{H}} \stackrel{\Phi_{-\mathbb{H}}^{-1}}{\longrightarrow} H^{*} B \mathrm{SU}(2)_{+} \stackrel{\Phi_{-\mathbb{H}_{\mathrm{ad}}}}{\longrightarrow} H^{*} B \mathrm{SU}(2)^{-\mathbb{H}_{\mathrm{ad}}}
$$

does not respect Steenrod operations. In general, a vector bundle $V \rightarrow X$ which is oriented with respect to a reasonable multiplicative cohomology theory $E^{*}$ defines a rank one projective $E^{*}(X)$-module $E^{*}\left(X^{V}\right)$, and thus an element of the Picard group of $E^{*}(X)$. These groups tend to be trivial, but their equivariant analogs (with respect to the cohomology automorphisms of $E$ ) can be more interesting.

The spherical fibration associated to $V$ defines a natural invariant

$$
\operatorname{Pic}_{\operatorname{Aut}(E)}\left(E^{*}(X)\right) \rightarrow H^{1}\left(\operatorname{Aut}(E),\left(1+\tilde{E}^{*}(X)\right)^{\times}\right)
$$

which can be pulled back to universal examples involving the $J$-groups of classifying spaces [6]. Techniques developed for the circle group [15] seem promising for $\mathrm{SU}(2)$ as well. 
2.2.4 Since this paper was submitted, J. Lurie's important work on topological field theories has become available. We close by drawing attention to some applications of his ideas to the subject of this paper.

Lurie's Theorem 2.5.10 [16] identifies the space of infinite-loop maps to an infinite loopspace $X=\left\{X_{n}\right\}$ from $|G|$ Cobord $\|\left.\right|^{+}$as the homotopy fixed-point spectrum $X^{h G}$ associated to an action of $G$ on $X$ via the natural action of $\operatorname{SO}(D)$ on suspension coordinates of the stabilization of $\Sigma^{D} X_{n-D}$. [This action is closely related to the constructions in the preceding paragraph.] The $n$th space of the fixed-point spectrum $X^{h G}$ is equivalent to the space of maps from the Thom spectrum $M T G(D)$ (in the notation of $\S 1.3 .1$ ) to $X_{n-D}$.

The infinite loopspace $B \otimes$ associated to the monoidal category of real vector spaces under tensor product is an interesting example. A monoidal functor from $G \mid$ Cobord| to $($ Vect, $\otimes$ ) is a generalization of a topological quantum field theory in Atiyah's sense, and it defines an infinite-loop map from $|G|$ Cobord $\|\left.\right|^{+}$to $B \otimes$, and hence an element of $k_{\otimes}^{-D}(M T G(D))$. These groups are accessible via the Atiyah-Segal exponential [5, 19]. 


\section{REFERENCES}

1. M.F. Atiyah, Thom complexes, Proc. London Math. Soc. 11 (1961) 291-310

2. —_ Riemann surfaces and spin structures, Ann. Sci. École Norm. Sup. 4 (1971) 47-62

3. — Topological quantum field theories, Inst. Hautes Études Sci. Publ. Math. No. 68 (1988), 175-186

4. ——, On framings of 3-manifolds, Topology 29 (1990) 1-7

5. — G.B. Segal, Exponential isomorphisms for $\lambda$-rings, Quart. J. Math. Oxford 22 (1971) 371-378.

6. - D.O. Tall, Group representations, $\lambda$-rings and the $J$-homomorphism. Topology 8 (1969) 253-297

7. C. Boyer, A note on hyper-Hermitian four-manifolds, Proc. Amer. Math. Soc. 102 (1988) 157-164

8. D. Ebin, The manifold of Riemannian metrics, in Global Analysis, Proc. Sympos. Pure Math., Vol. XV pp. 11-40, AMS (1970)

9. S. Galatius, I. Madsen, M.Tillmann, M.Weiss, The homotopy type of the cobordism category, available at math. AT/0605249

10. E. Getzler, The Toda conjecture, in Symplectic geometry and mirror symmetry (Seoul, 2000), 51-79, World Sci. Publ.(2001), available at arXiv:math/0108108

11. J. Giansiracusa, The stable mapping class group of simply connected 4-manifolds, J. Reine Angew. Math. 617 (2008) 215-235, available at math.GT/0510599

12. P. Goerss, Hopf rings, Dieudonné modules, and $\Omega^{2} S^{3}$, in Homotopy invariant algebraic structures 115-174, Contemp. Math. 239, AMS (1999)

13. R. Gompf, A. Stipsicz, 4-manifolds and Kirby calculus, AMS Grad. Studies in Mathematics 20 (1999)

14. L. Hesselholt, I. Madsen, The $S^{1}$-Tate spectrum for $J$. Papers in honor of Jos Adem, Bol. Soc. Mat. Mexicana (2) 37 (1992) 215-240

15. R. Kirby, P. Melvin, Canonical framings for three-manifolds, in Proceedings of 6th Gökova Geometry-Topology Conference, Turkish J. Math. 23 (1999) 89-115, available at math.GT/9903056

16. J. Lurie, On the classification of topological field theories, available at http://math.mit.edu/ lurie/

17. J. Milnor, Lectures on the $h$-cobordism theorem, Princeton (1965)

18. J. Morava, Heisenberg groups in algebraic topology, in Topology, geometry and quantum field theory 235-246, London Math. Soc. Lecture Notes 308, Cambridge (2004), available at math. AT/0305250

19. C. Rezk, The units of a ring spectrum and a logarithmic cohomology operation, J. Amer. Math. Soc. 19 (2006) 969-1014, available at arXiv:math/0407022

20. G. Segal, Categories and cohomology theories, Topology 13 (1974) 293-312

21. - The definition of conformal field theory, in Topology, geometry and quantum field theory 421-577, London Math. Soc. Lecture Notes 308, Cambridge (2004) 
22. L. Taylor, Complex spin structures on 3-manifolds, in Geometry and topology of manifolds 313-317, Fields Inst. Commun. 47, AMS (2005)

Jack Morava

Johns Hopkins University, Baltimore, Maryland

E-mail: jack@math.jhu.edu

Nitu Kitchloo

University of California at San Diego, San Diego, California

E-mail: nitu@math.ucsd.edu 